\title{
Design and Development of Detector Simulator for Total Ionized Dose and ground checkout system of radiation monitoring instrument
}

\author{
Keyur Mahant, Chintan Bhatt
}

\begin{abstract}
This Paper describes the simulator development for the Total Ionizing Dose (TID) measurement of radiation monitoring instrument. The TID Detector (UDOS001-micro dosimeter) is a compact hybrid microcircuit which directly measures Total Ionizing Dose absorbed by an internal silicon test mass. The developed detector simulator, simulates the equivalent Total Ionized Dose absorbed from the space radiation and Ground checkout simulator receive the data from Radiation monitoring Instrument through UART and process it for the functional verification of the Radiation monitoring Instrument, which is discussed in the paper.
\end{abstract}

\section{Keywords — Radiation, Total Ionizing Dose, LabVIEW}

\section{INTRODUCTION}

$\mathbf{T}$ HERadiation is energy that comes from a supply and travels through space and should be able to penetrate numerous materials [1]. Light, radio, and microwaves are varieties of radiation that are known as non-ionizing. The sort of radiation mentioned during this document is named ionizing radiation as a result of it will turn out charged particles (ions) in matter. Ionizing Radiation is made by unstable atoms. Unstable atoms disagree from stable atoms as a result of unstable atoms have excess of energy or mass or each. Radiation also can be made by high-voltage devices. Atoms with unstable nuclei are said to be radioactive. So as to achieve stability, these atoms give off, or emit, the surplus energy or mass. These emissions are called radiation. The sorts of radiation are electromagnetic and particulate (i.e., mass given off with the energy of motion). Gamma radiation and $\mathrm{x}$ rays are samples of electromagnetic radiation. Gamma radiation originates within the nucleus while $\mathrm{x}$ rays come from the electronic part of the atom. Beta and alpha radiation are samples of particulate radiation. Curiously, there is a "background" of natural radiation all over in our environment. Ubiquitous background radiation comes from space (i.e., cosmic rays) and from naturally occurring radioactive materials contained within the earth and in living things. Space radiation consists of particles trapped within the Earth's magnetic field, Particles shot into space during solar flares (solar particle events) and Galactic cosmic rays, which are high-energy protons and substantial ions from outside our

This work was supported by CHARUSAT Space \& Research Technology Center.

Chintan Bhatt and Keyur Mahant are with Charotar University of Science And Technology (e-mail: chintanbhatt.ce@charusat.ac.in, keyurmahant.ec @ charusat.ac.in). scheme [2]. Solar Flares additionally contribute varying quantities of electrons, protons, and lower energy substantial ions.

When these radiations comes into the contact with electronics it causes Non-destructive or destructive damages to the electronics like Heavy ions of various energies cause single event effects (SEE) [3]. Non-destructive or "soft-error effects" momentarily or permanently change the state of a device or cell/node, affecting its functionality [3]. Total Ionized Dose (TID) effect is accumulation of ionizing dose deposition over a long time on electronics. The TID, largely due to protons and electrons, can result in device failure. TID will be measured in terms of the absorbed dose, which is a measure of the energy absorbed by matter [4]. Absorbed dose is quantified using a unit called the RAD. Here, UDOS001 is used as TID detector for the measurement of absorbed radiation dose.

\section{BACKGROUND}

\section{A. Standard Radiation Environment Monitor (SREM) [10]}

A decision was done by the ESA management board to develop and fly a Regular Radiation Environment Monitor on future ESA projects with the subsequent objectives [9]:

- To assist satellite performance, anomaly and failure investigations.

- With preloaded radiation thresholds to act as a warning / warning arrangement for the spacecraft.

- To assist within the improvement of design of future spacecraft.

- To permit the creation and maintenance of a European radiation database.

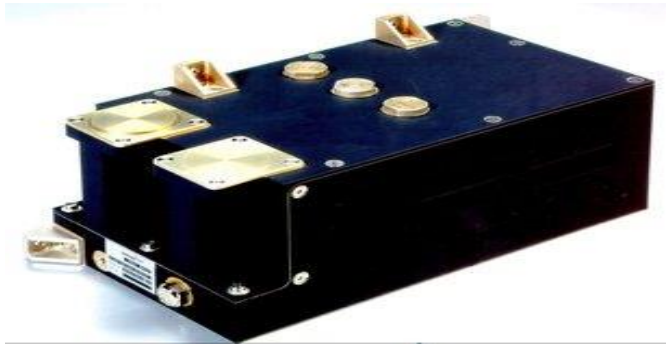

Fig. 1. SREM Model

SREM is an enhanced version of a previous Radiation Environment Monitor (REM) flown on the MIR space station and the micro-satellite STRV. The first SREM flight is planned on STRV1-C. The SREM energy resolution for electrons is 
between about $300 \mathrm{keV}$ and $7 \mathrm{MeV}$. For protons the energy resolution is about between $10 \mathrm{MeV}$ and hundreds of $\mathrm{MeV}$.

\section{B. Proton Monitor (PM) [10]}

ESA recognized the requirement for a low-mass, low power proton monitor to be used on spacecraft where the trapped proton radiation belts or solar flares are of specific concern. Supported this it had been determined to support the adaptation of a terrestrial Radon monitor (developed by Thomson \& Nielsen Electronics Ltd.) which has shown to possess the essential characteristics for a proton monitor.

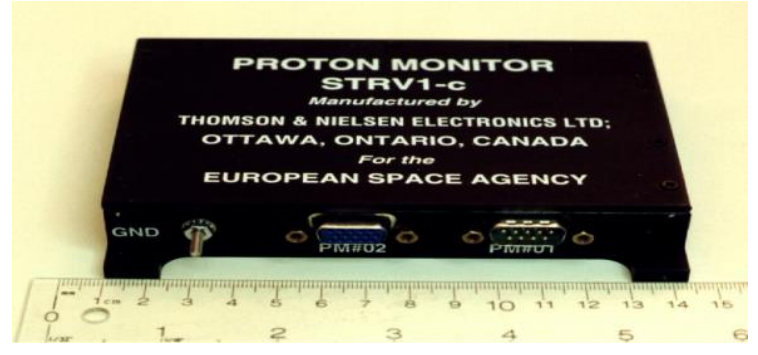

Fig. 2. Proton Monitor (PM) model

Following demonstrates the proton monitor:

- The dimensions of the PM are $135 \mathrm{~mm} \times 100 \mathrm{~mm} \times$ $25 \mathrm{~mm}$.

- The PM power consumption is $300 \mathrm{~mW}$.

- $\quad \mathrm{PM}$ is powered by $12 \mathrm{~V}$ to $40 \mathrm{~V}$.

- The PM mass is $350 \mathrm{~g}$.

\section{Cosmic Ray Telescope for the Effects of} Radiation (CRaTER) [10]

Among those instruments is CRaTER Cosmic Ray Telescope for the Effects of Radiation. CRaTER characterizes the worldwide lunar radiation environment and its biological impacts by measuring galactic and solar cosmic ray radiation behind a "human tissue-equivalent" plastic.

LRO's science payload of seven instruments gathers statistics beneficial to additional exploration of the moon. Among those instruments is CRaTER Cosmic Ray Telescope for the Effects of Radiation.

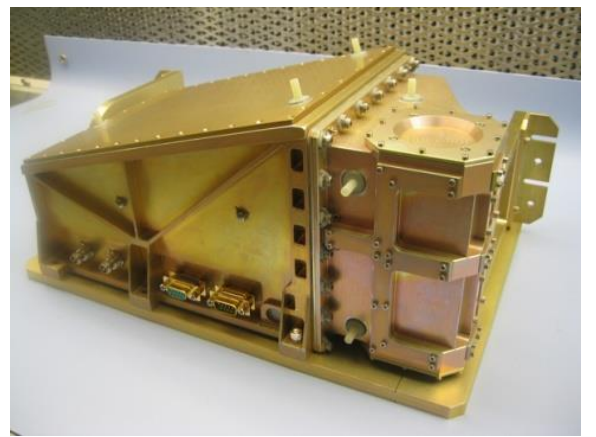

Fig. 3. CRaTER model

CRaTER examination goals:

- Measure and characterize the deep space radiation environment in terms of LET spectra of galactic and solar cosmic rays (particularly beyond $10 \mathrm{MeV}$ ).

- Mass is $5.53 \mathrm{~kg}$.

- Power is $6.66 \mathrm{~W}$.

\section{WORKING OF DOSIMETER AND SIMULATOR}

The UDOS001-micro dosimeter incorporates a silicon detector and a pulse-processing design that makes a Gaussian shaped pulse in response to ionizing radiation. The Gaussian pulse is given to an integrator that integrates the area under the pulse. In this way pileup effects are reduced. The integration lasts for each event till a predetermined limit is reached. A Quanta of charge is detached from the integrator equivalent to a rate of 14urads, and a counter value is incremented. This counter value is separated into sub-groups of 8 bits which are each presented to a D/A converter. The DAC LOW range is offeres dose as 14 urads per $19.5 \mathrm{mV}$ steps, the DAC MED range is 256 times the LOW range, and the HIGH is 256 times the MED range. The UDOS001-micro dosimeter can retain the value of the dose for as long as it is powered. A Pseudo-LOG output is commonly sampled at awfully low rate to observe the total dose over extended periods of time.The other DAC ranges is sampled at higher rates to obtained handy dose rate measurements.

The Total ionizing dose (TID) [11], largely owing to electrons and protons, may end up in device failure. In either case, TID is measured in terms of the absorbed dose that could be a measure of the energy absorbed by matter. Absorbed dose is quantified using either a unit called the RAD or the SI unit which is the gray (Gy).

$$
1 \mathrm{~Gy}=100 \mathrm{rads}=1 \mathrm{~J} / \mathrm{kg}
$$

The TID is calculated from the protons and electrons. The key sources of the protons and electrons are:

- Solar energetic particle events (from solar flares).

- Passage through the South Atlantic Anomaly.

In low earth orbit (LEO), the foremost dose supply is from electrons and protons (inner belt); and in geostationary earth orbit (GEO), the prime supply is from electrons (outer belt) and solar protons. The full accumulated dose depends on orbit altitude, orientation, and time. To compute TID, we want to know the integrated particle energy spectrum, (E), that is, fluence as a function of particle energy. Satellites naturally encounter TID up to $10 \mathrm{krad}$ (Si) in LEO. As TID increases, materials degradation will increase, for instance, solar cell output can decrease. Long-term exposure will cause device threshold shifts, augmented device leakage and power consumption, timing changes, and decreased functionality. TID effects could also be lessened using radiation hardened devices and shielding. Electrons and low energy protons can also be partly lessened with shielding. Since each TID and SEE (single event effects) are from ionizing radiation, it is important to address the difference among the two with respect to design and analysis. TID could be a long-term failure mechanism versus SEE that is an immediate failure mechanism. TID Detector simulator generates data similar to UDOS001 (LOW, MED, HIGH and LOG) in the range of 0-5V. In TID detector simulator value Total Ionization Dose can be entered into various formats such as RAD, mili-RAD and micro-RAD, which will be further, converted into LOW, MED and HIGH by the simulator using mathematicaloperations. To generate the equivalent value of LOG datasheet from Teledyne was used, which was called into the simulator from excel sheet. Moreover, before transmitting these data to R-C network comparison is done between LOG data and LOW, MED \& HIGH data for the simulator verification. 
Binary values of LOW, MED, HIGH and LOG are given to second order R-C network using UART to generate equivalent voltage as of UDOS001.

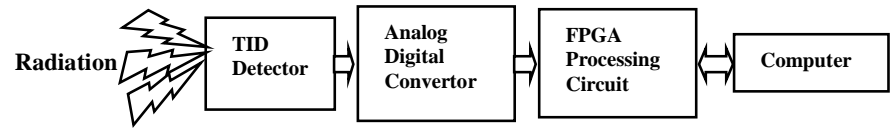

Fig. 4. System block diagram of TID measurement system

Tentative Power, Volume, Weight Budget of TID (Total Ionizing Dose):

- Power $200 \mathrm{~mW}$.

- Dimensions 70X70X50 mm.

- Weight $400 \mathrm{gm}$.

Ground checkout system for Radiation monitoring Instrument is used for functional verification and validation of the instrument. Ground checkout system receives the data in the form of a string. String contains LOW, MED, HIGH, LOG, RESET and TIME TAG. String is defragmented later on. Comparison is done in between sum of LOW, MED \& HIGH and LOG. Value of LOG is obtained from the LUT. LUT for LOG data is made with the help of datasheet of Teledyne. Individual values of LOW, MED, HIGH, RESET \& LOG and DIFF displayed on front panel along with TIME TAG. DIFF is difference between sum of LOW, MED, HIGH \& RESET and LOG.

\section{A. Subsystems of TID (Total ionizing dose)}

I) Detector - Micro Dosimeter ( $\mu$ DOS001)

II) Front end Electronics

III) Back end Electronics

Micro Dosimeter Features ( $\mu$ DOS001):

$\begin{array}{ll}\text { Size } & 1.4 \text { " x } 1.0 " \text { " } 0.040 " \\ \text { Weight } & 20 \text { grams } \\ \text { Performance } & 10 \mathrm{~mA} \text { from } 13 \mathrm{~V} \text { to } 40 \mathrm{~V} \text { input } \\ & 14 \mu \mathrm{rads} \text { to } 40 \mathrm{Krads}\end{array}$

Working principle of Radiation Micro Dosimeter ( $\mu$ DOSO01):

- The Micro Dosimeter ( $\mathrm{P} / \mathrm{N} \mu \mathrm{DOS} 001)$ is a compact hybrid microcircuit which directly measures total ionizing dose (TID) absorbed by an internal silicon test mass.

- The dosimeter provides output in four channels (LOW, MED, HIGH, and LOG). The LOW, MED, and HIGH each represent a progressively more significant byte, scaled to 0 to $5 \mathrm{~V}$.

- The accumulated dose is presented to three DC linear outputs and one pseudo-logarithmic output giving a dose resolution of 14 uRads and a measurement range up to 40 krads.

- These outputs are intended to be directly connected to most analog-to-digital converters (ADCs).
Heritage of Micro Dosimeter Missions ( $\mu$ DOS001):

- 2009: NASA Lunar Reconnaissance Orbiter.

- 2009: NASA International Space Station MISSE7B test bed.

- 2009: NRL \& Boeing, LEO orbit.

\section{Front end and Back end Electronics:}

Radiation is measure by Detector/Dosimeter that data will provide to Analog Processing Circuit and FPGA for further processing. Using processed data we can measure the Total Ionizing Dose available in the space.

\section{Data rate Calculation of TID Measurement:}

DOSE RESOLUTION/COUNT: $14 \mu \mathrm{rad}$

TOTAL DOSE MEASURE/COUNTER: $235 \mathrm{rad}$

MEMORY SPECIFICATION: Memory required $=60$ bits

\section{DATA RATE:}

Required Minimum Data/hr $=60 \mathrm{bits} / \mathrm{hr}$.

Data should be transmitted $/$ day $=24 * 60=1440$ bits $/$ day.

Transmission time from LEO to ground $=10$ mins.

So minimum data rate $=1440 / 600=2.4 \mathrm{bit} / \mathrm{sec}$.

Minimum Clock Frequency Require $16 \mathrm{~Hz}$.

\section{ALGORITHM}

\section{B. Detector Simulator for TID}

In the TID detector simulator, values of the radiation can be entered in the form of RAD. Initially comparison of RAD value is done. If RAD value is greater than $235 \mathrm{RAD}$ value of reset counter is incremented.Once the comparison is done, further value of RAD is converted into LOW, MED, HIGH and LOG by doing mathematical operations.After that frame will be generated, which is having LOW, MED, HIGH, LOG, RESET and TIME TAG.TIME TAG is taken from the system. Frame is the size of 48 bits. The frame is sent in the string format by UART to the instrument using LabVIEW software.The generated frame is shown in the table I.

Table. I

Data frame of Detector Simulator for TID

\begin{tabular}{|lll|lll|l|l|l|}
\hline LOW & \multicolumn{2}{|c|}{ MED } & HIGH & LOG & RESET & TIME TAG \\
\hline 0 & 8 & 16 & 24 & 32 & $\mathbf{4 0}$ & $\mathbf{4 8}$ (bits) & &
\end{tabular}

Here, the 8 bit DAC is used because the DAC inside the dosimeter is of 8 bit. The range of DAC is 0 to 255 and the reference voltage level is from $0-5 \mathrm{Volts}$. The corresponding voltage values of LOW, MED, HIGH \& LOG are generated by following equation.

$$
\text { Voltage }=\frac{5}{256} * \mathrm{X}
$$

Where, $\mathrm{X}$ is the Decimal value of the LOW, MED, HIGH \& LOG. The Data frame generated from the simulator simulates the same output with Radiation monitoring Instrument as the Detector generates the output. Output (LOW, MID, HIGH and $\mathrm{LOG})$ is in the range of $0-5$ Volts. 


\section{Ground Checkout Simulator for TID}

The generated frame form the instrument is received in LabVIEW using UART protocol. Received frame is of 48 bits in the string format.The received data string is defragmented into byte array and this byte array is divided into LOW, MED, HIGH, LOG, RESET and TIME TAG. From the obtained data packet in the form of byte, the sum of radiation is calculatedby using mathematical operations.As discussed in the Detector simulator, 8 bit DAC is usedbecause the DAC inside the dosimeter is of 8 bit.Also, if concatenate the LOW, MED \& HIGH data packet, frame size will be 24 bits. This data packet will give the output up to $235 \mathrm{RAD}$ and again it will start from 0 . The Detector (UDOS001-micro dosimeter) range is 040KRAD. So for the above range of 235RAD, there is separate 8 bit Reset counter will increasing accordingly. The sum of LOW, MED, HIGH and RESET data packets will generate the RAD value, which will defragment the receive data frame to data packets. Also the mathematical calculation is shown below for converting byte array data packets conversion to RAD. An example is shown in the following figure 5.

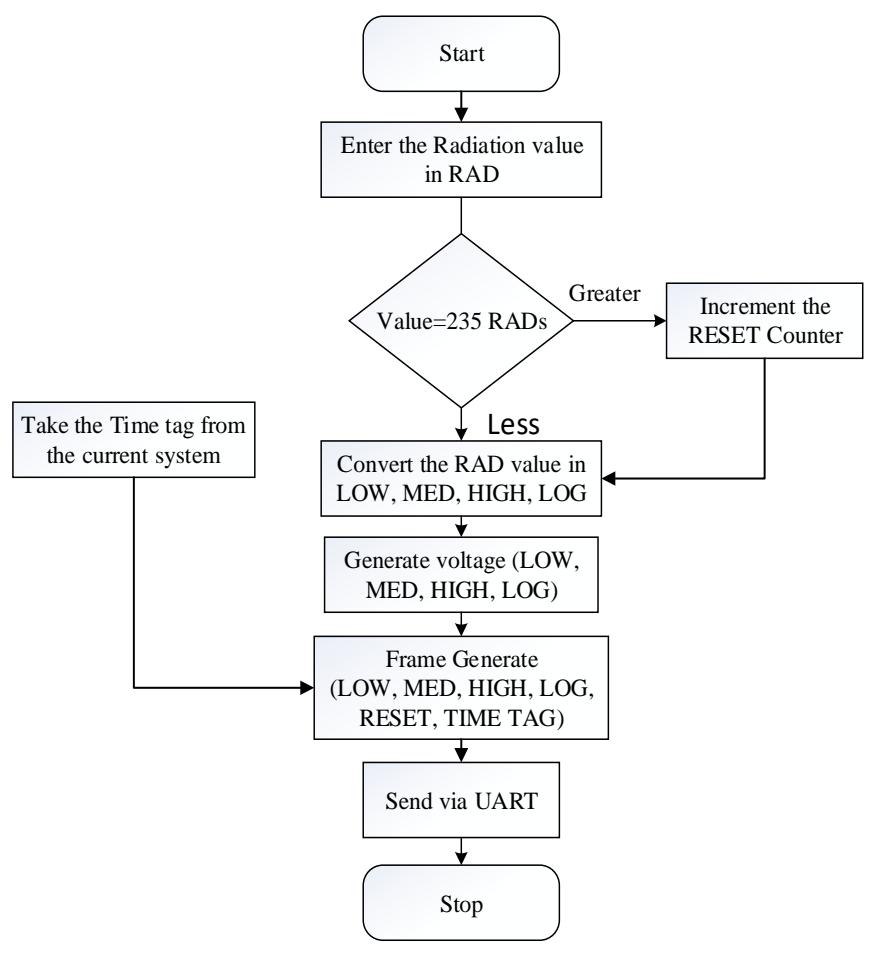

Fig. 5. Flowchart of Detector Simulator for TID
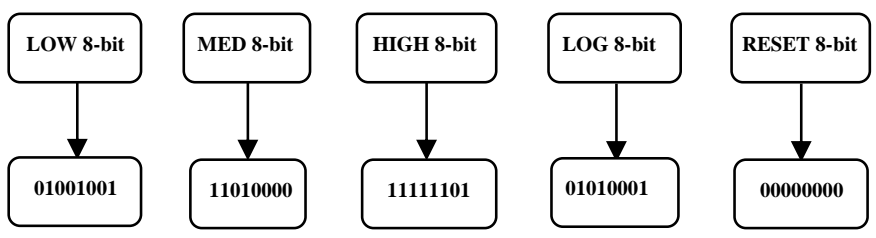

Fig. 6. Data frame defragmentation of Ground Checkout Simulator for TID

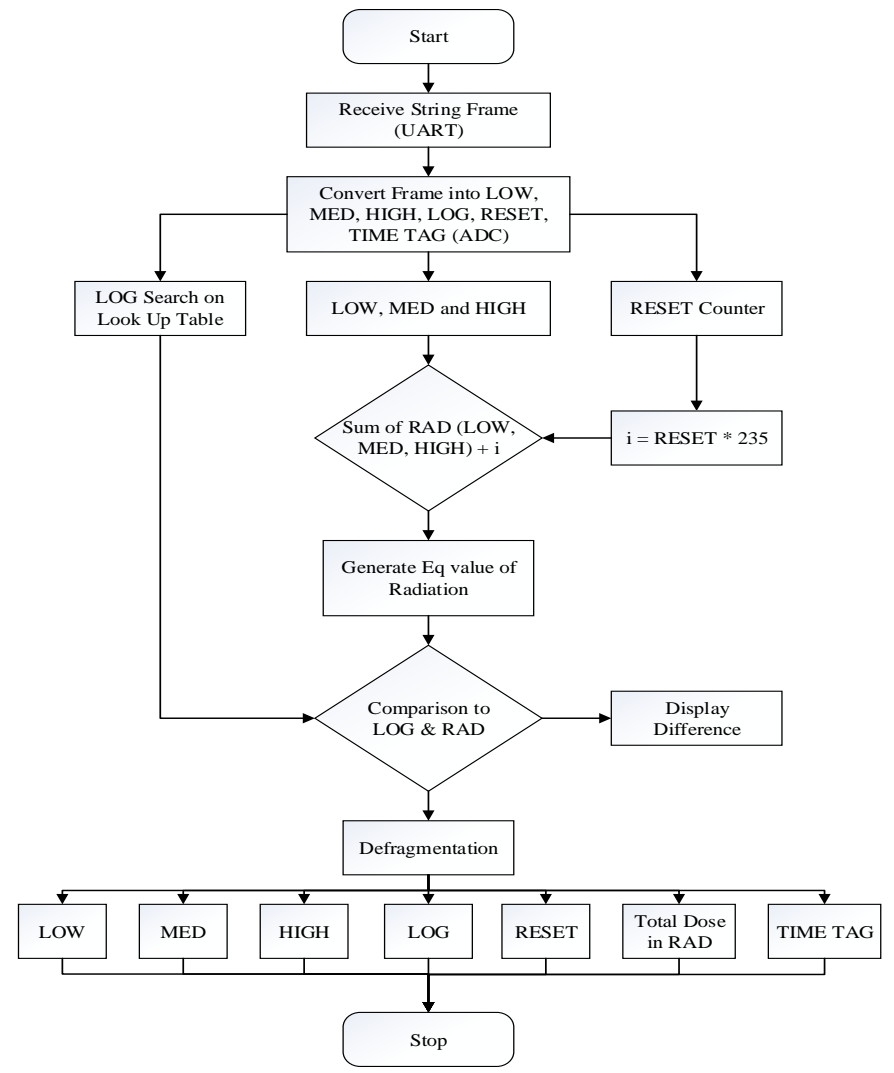

Fig. 7. Flowchart of Ground Checkout Simulator for TID

LOW - $0 \begin{array}{llllllll}1 & 0 & 0 & 1 & 0 & 0 & 1\end{array}$

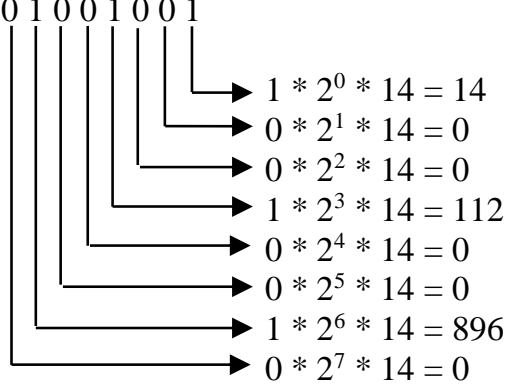

LOW $($ sum of radiation $)=1022$ micro-RAD

$$
=0.001022 \mathrm{RAD}
$$

MED - 11100100000

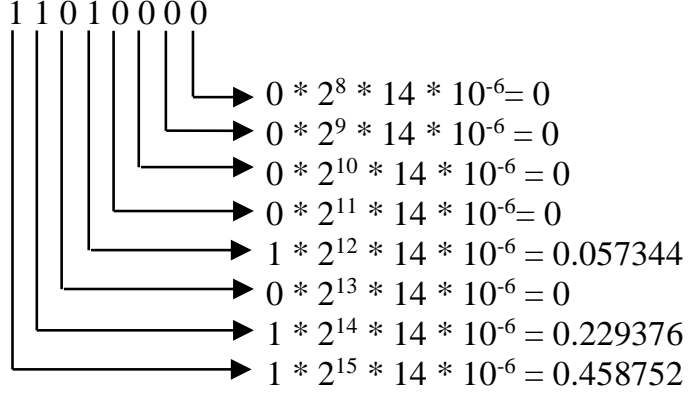

$\mathrm{MED}($ sum of radiation $)=0.745472 \mathrm{RAD}$ 


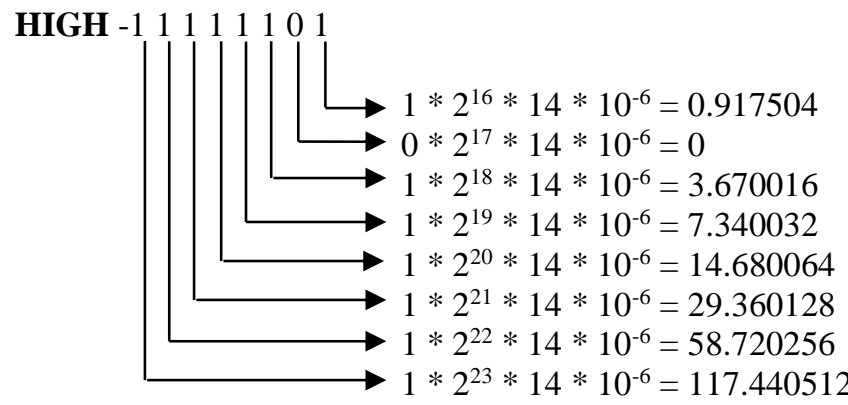

$\mathrm{HIGH}($ sum of radiation $)=232.128512 \mathrm{RAD}$

$$
\begin{aligned}
& \text { Sum of Radiation }(\mathrm{LOW}+\mathrm{MED}+\mathrm{HIGH}) \\
& \quad=0.001022+0.745472+232.128512 \\
& =\mathbf{2 3 2 . 8 7 5 0 0 6 R A D}
\end{aligned}
$$

After doing mathematical operation, convert the byte data of LOG to equivalent decimal value. The decimal value of LOG is converted in terms of voltage as shown in equation (3).

LOG - 01010001 = 81 (decimal value)

Convert into voltage $=\frac{5}{256} \times 81=1.582 \mathrm{~V}$

From the obtained value simulator will retrieve the LOG value from the LUT. Here, value of voltage is 1.582 and as per the LUT, value of LOG is 234.88 RAD which is very near to the value obtained from LOW, MID, HIGH and LOG counter. Search on Look up,

$$
\begin{aligned}
& \log \mathrm{DAC}(\mathrm{V})-1.582 \\
& \text { Dose(RAD) - } 234.88
\end{aligned}
$$

LUT is made from the datasheet given by Teledyne, the RAD value can be determined. This RAD value is approximate of the RAD value generated by detector simulator.Sum of RAD would be equal or nearer to the RAD value obtained from the LOG value. Then compare the RAD value obtained from of LOG and Equivalent value of RAD obtained by LOW, MED, $\mathrm{HIGH}$, and RESET. Here, the difference in the two obtained value is 2.004994. After all data defragmented in from of LOW, MED, HIGH, LOG, RESET, Total Dose in RAD and TIME TAG.The difference is shown by the following example.

\section{Difference-}

Difference $=$ Dose $($ RAD) obtained by LOG-Radiation Dose (LOW+MED+HIGH+ Reset)
$=234.88-232.875006$

$$
\text { = 2.004994 RAD }
$$

\section{SOFTWARE DETAILS}

Laboratory Virtual Instrumentation Engineering Workbench (LabVIEW) is a platform and development environment for the visual programming language. LabVIEW software is developed by the National Instruments. LabVIEW is commonly used for data acquisition, instrument control, and industrial automation by using visual programming [5]. LabVIEW is used for data acquisition in the Detector Simulator for TID and Ground checkout simulator for TID. LabVIEW have different function blocks, which are used in the TID Detector\& Ground checkout simulator such as structure, array, numeric, string, comparison, Boolean functions, timing, UART, Loop etc.

The Universal Asynchronous Receiver/Transmitter (UART) controller is the key factor of the serial communications. UART is a serial communication protocol. The UART receipts bytes of data and transmits the individual bits in a successive sequence. At the destination, UART re-assembles the bits into complete bytes. Transmitting and receiving UARTs must be set at the equal baud rate, character length, parity, and stop bits for correct operation. The usual format for serial ports used with PC connected to modems is 1 Start bit, 8 data bits, no Parity and 1 Stop bit [6]. Here the UART baud rate is 9600 .

\section{EXPERIMENT RESULTS}

Detector Simulator and Ground Checkout Simulator is used for the radiation measurement. The simulator is created using LabVIEW software. Taking Radiation value input in the form of RAD. After that RAD value converted into LOW, MED, HIGH \& LOG. LOG is generated from .XLS, which is taken from Look up Table. These values are transferred through UART with band rate of 9600 . These values will be further converted into voltage using second order RC filter. Moreover simulator display the values of the equivalent voltage for a particular RAD values.Simulator receiving the data in the form

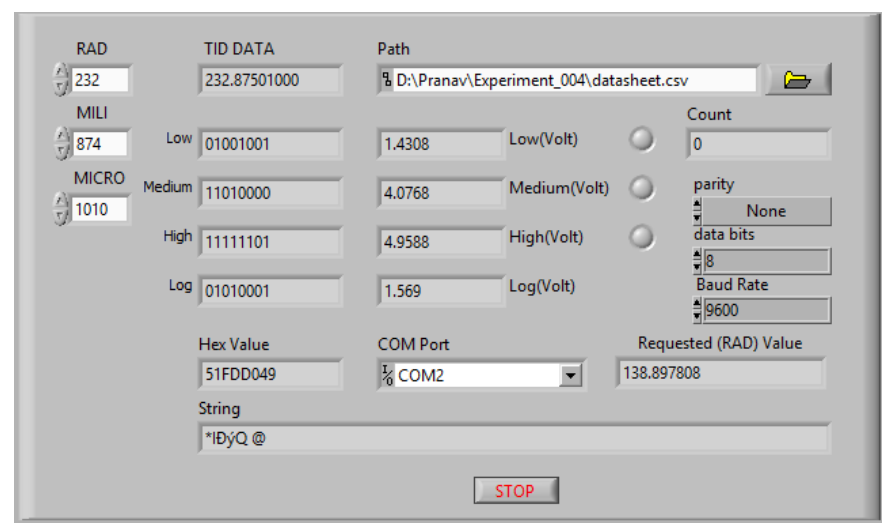

Fig. 8. Detector Simulator for TID

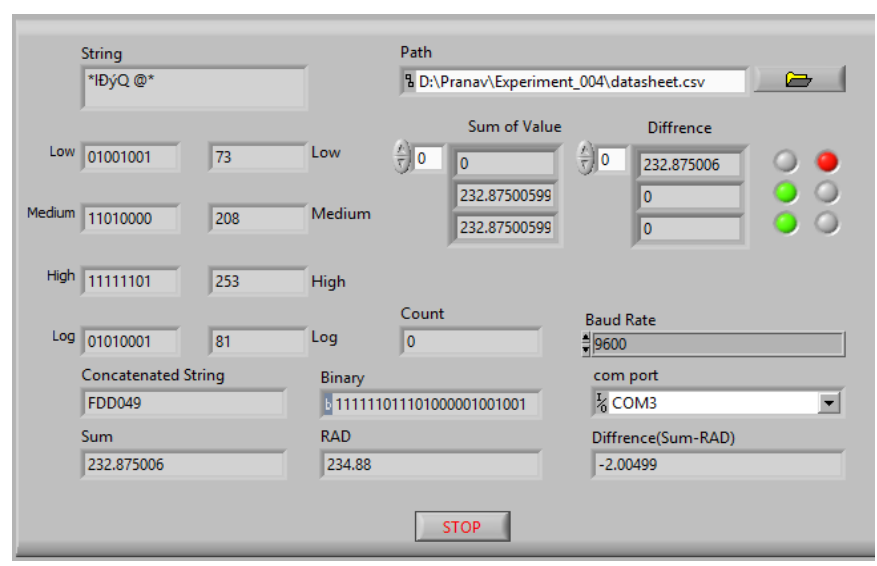

Fig. 9. Ground Checkout Simulator for TID 
of data packets. Which is defragmented later on. After defragmentation we are sorting the values in terms of LOW, MED, HIGH and LOG. Apart from LOW, MED, HIGH and LOG additional Reset counter value is received. Which is required for the measurement of Radiation value more then 235RADs.

\section{CONCLUSIONS}

In this paper, developed Simulator for Detector is generalized simulator for the dosimeter UDOS001 which can be useful for all the dosimeter users and it is also well calibrated and tested. Ground Checkout System Simulator is hardware specific for our instrument testing and functional verification. It is used for measuring the absorbed dose (TID) by the dosimeter. Moreover, Developed simulator can be veryuseful for the many other data acquisition system data processing by implementing minor changes.

\section{FUTURE SCOPE}

The Ground checkout system currently uses the UART protocol for receiving data. In the future, For more data transfer rate (around $11 \mathrm{Mbps}$ speed) with secure data transfer, TCP/IP protocol can be used and implemented. Also the web application can be implemented for Remotly monitoring the data recived from the instrument. GPS location can be use for receiveing the information from space, The software can also show the geo- graphical locations of the satellite carring the instrument with Radiation dose rate at perticular location with time-stamp.

\section{REFERENCES}

[1] Radiation Emergency Assistance Center,"Guidance for radiation accedent management", available at www.orise.orau.gov/reacts/guide/ define.htm

[2] Lyndon B. Johnson Space Center, "Understanding Space Radiation", National Aeronautics and Space Administration, October 2002

[3] Johnson Space Center (JSC), "SPACE RADIATION EFFECTSON ELECTRONIC COMPONENTSIN LOW-EARTH ORBIT", National Aeronautics andSpace Administration, April 1996

[4] Mazur, J. E., W. R. Crain, M. D. Looper, D. J. Mabry, J. B. Blake, A. W. Case, M. J. Golightly, H. E. Spence, andJ. C. Kasper, "Newmeasurements of total ionizing dose in the lunar environment",S07002, doi:10.1029/2010SW000641, Space Weather, 2011

[5] Guangying Yang, "The Simulation Application of LabVIEW in the DataProcessing", 978-1-61284-459-6/11/\$26.00 @2011 IEEE

[6] F. Baronti, A. Lazzeri, R. Roncella, R. Saletti, Universita di Pisa, "Firmware/Software Platform for Rapid Development of PC-based Data Acquisition Systems",ICECS , 978-1-4244-8157 -6/1 0/\$26.00 @20 10 IEEE

[7] Mohindar Vaghela, Rachna jani, Keyur Mahant, "FPGA Implementation of Digital pulse processing techniques for Radiation Measurement".

[8] Pavel Fexa, Josef Vedral, "Developing Automated Data Acquisition Systemfor ADC and DAC Testing", International Conference on Intelligent Data Acquisition and Advanced Computing Systems, 978-14577-1425-2/11/\$26.00 @2011 IEEE

[9] Microelectronic Technologies UDOS001 Micro Dosimeter.

[10] New measurements of total ionizing dose in the lunar environment.

[11] Case, A. W., H. E. Spence, M. J. Golightly, J. C. Kasper, J. B. Blake, J. E. Mazur, L. W. Townsend, and C. J. Zeitlin (2010), GCR access to the Moon as measured by the CRaTER instrument on LRO, Geophys. Res. Lett., 37, L19101, doi:10.1029/2010GL045118. 